\title{
Influence of Body-Implanted Capsule Dimensions and Materials on Achievable Radiation Efficiency
}

\author{
Denys Nikolayev \\ $M A G-S C I-S T I-A S$ \\ EPFL \\ Lausanne, Switzerland \\ d@deniq.com
}

\author{
Maxim Zhadobov \\ IETR - UMR CNRS 6164 \\ Université de Rennes 1 \\ Rennes, France \\ Maxim.Zhadobov@univ-rennes1.fr
}

\author{
Wout Joseph \\ INTEC - WAVES \\ imec / Ghent University \\ Ghent, Belgium \\ Wout.Joseph@ugent.be
}

\author{
Luc Martens \\ INTEC - WAVES \\ imec / Ghent University \\ Ghent, Belgium \\ Luc1.Martens@ugent.be
}

\author{
Ronan Sauleau \\ IETR - UMR CNRS 6164 \\ Université de Rennes 1 \\ Rennes, France \\ Ronan.Sauleau@univ-rennes1.fr
}

Anja Skrivervik
$M A G-S C I-S T I-A S$
EPFL

Lausanne, Switzerland

Anja.Skrivervik@epfl.ch

\begin{abstract}
Fundamental bounds on achievable radiation efficiency serve as the design quality gauge, facilitate the choice of the antenna type considering the available dimensions, and provide simple rules to check the feasibility of a given design. This study quantifies the effect of body-implanted capsule dimensions and materials on achievable radiation efficiency. We also show that a dielectric-loaded electric antenna operating close to the optimal frequency can radiate more efficiently than a magnetic one. The latter, however, is more efficient when electrically small.
\end{abstract}

Keywords-conformal antenna, dielectric loading, electric antenna, implantable, magnetic antenna, radiation efficiency

\section{INTRODUCTION}

Wireless body-implanted encapsulated devices have many potential applications in medicine, clinical research, professional sports, occupational health, and defense [1]-[3]. These capsules commonly use radiofrequency (RF) antennas to communicate with external on- or off-body systems. A wide range of RF antennas has been proposed for body-implantable applications [4]-[15]. Yet, establishing robust links between an in-body capsule and external equipment remains a major challenge because of too low efficiencies $(<0.1 \%)$ of the antennas operating in lossy media with uncertain electromagnetic (EM) properties [16], [17]. Considering typical maximum input power levels ranging from a few to about $50 \mathrm{~mW}$ [18] (limited by safety standards) and Rx sensitivities, this efficiency provides an operating range up to only a few meters [19]. Therefore, fundamental studies are required to investigate ways of improving the efficiency of body-implanted antennas. Impact of tissues on radiation performance has been considered in [20] for small inductor sources and in [16], [21] for infinitesimal magnetic and electric sources. Finite-sized $\mathrm{TM}_{10}$ and $\mathrm{TE}_{10}$ sources were considered in [17]. In this study, we evaluate the effects of the antenna dimensions and surrounding capsule materials on achievable radiation efficiencies in the $0.1-4-\mathrm{GHz}$ range.

This publication is supported by the European Union through the European Regional Development Fund (ERDF), and by the French region of Brittany, Ministry of Higher Education and Research, Rennes Métropole and Conseil Départemental 35, through the CPER SOPHIE/STIC \& Ondes.

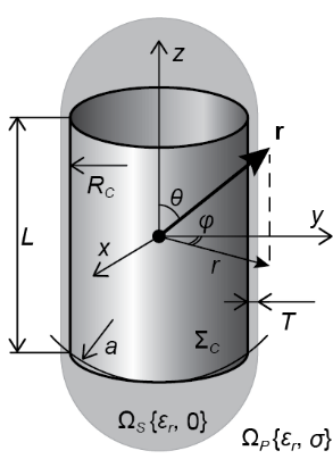

Fig. 1. Problem formulation. Current density distributions on a cylindrical surface $\Sigma_{C}$ define the source surrounded by a losses capsule-shaped volume $\Omega_{S}$. The capsule is centered inside of a $\varnothing 100$-mm spherical phantom $\Omega_{P}$ (not shown) with muscle-equivalent EM properties.

\section{PROBLEM FORMULATION}

An equivalent source representing a body-implanted capsule antenna is centered inside of a $\varnothing 100-\mathrm{mm}$ spherical phantom with dispersive muscle-equivalent EM properties according to [22]. The source (Fig. 1) is defined as a current density $\mathbf{J}_{s}$ distribution on a parametrized cylindrical surface $\Sigma_{C}$ surrounded by a lossless (i.e. $\sigma=0$ ) capsule-shaped volume $\Omega_{S}$.

Two sources are considered: 1) an "electric" $\mathrm{TM}_{10}$ defined as $\mathbf{J}_{s}=[0,0, \cos (\pi z / L)]$ and 2$)$ a "magnetic" $\mathrm{TE}_{10}$ defined as $\mathbf{J}_{s}=(0,1,0)$. The radiation efficiency is obtained using the Poynting's theorem [23] as $\eta \equiv \operatorname{Re}\left(P_{e}\right) / \operatorname{Re}\left(P_{s}\right)$, where the exiting power $P_{e}$ and the supplied power $P_{s}$ are evaluated as in [17].

Considering the $z$-axial symmetry of the formulated problem (Fig. 1), it can be reduced to $\mathbf{R}^{2}$ assuming $\mathbf{E}(r, \varphi, z)=$ $\overline{\mathbf{E}}(r, z) e^{-j m \varphi}$, where $m$ is the azimuthal mode number. Now, the solution of the governing inhomogeneous time-harmonic wave equation can be obtained numerically using, for instance, a fully-adaptive $h p$-FEM (finite element method) [24]. 


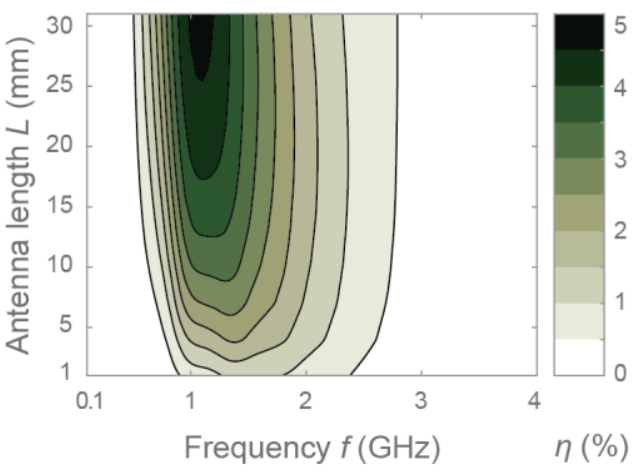

(a)

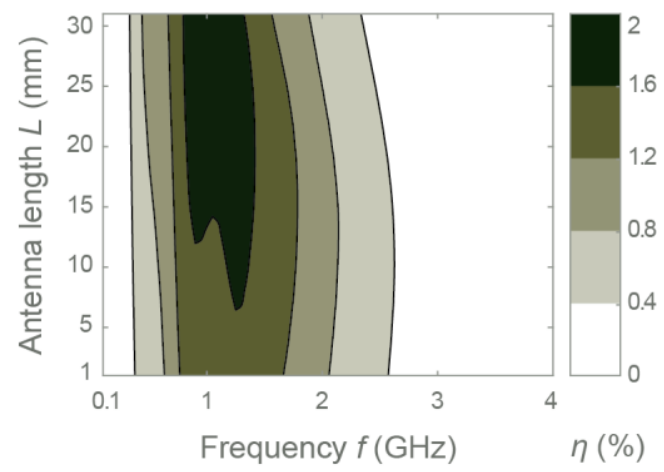

(b)

Fig. 3. Achievable radiation efficiencies of a capsule antenna operating at (a) $\mathrm{TM}_{10}$ mode and (b) $\mathrm{TE}_{10}$ mode as a function of the antenna length $L$. A $\varnothing 100-\mathrm{mm}$ spherical muscle-equivalent phantom accounts for losses in biological tissues.

\section{III.RESULTS}

The radiation efficiency $\eta$ follows a skew-normal distribution with its peak defined as an optimal operating frequency $f_{\text {opt }}$. Both $\eta$ and $f_{\text {opt }}$ strongly depend on the phantom formulation (addressed previously in [21], [17]) and the source parameters. As for the latter, the physical length of the source has the strongest effect on achievable radiation performance. Fig. 2 shows the effect of the antenna length $L$ on $\eta$ and $f_{\text {opt }}$ computed in the $\varnothing 100$-mm spherical phantom with muscleequivalent EM properties. For $\mathrm{TM}_{10}$, the longer the source is, the higher the $\max (\eta)$. The optimal frequency is inversely proportional to the length. In contrast, for $\mathrm{TE}_{10}$ the efficiency peaks around $1.5 R_{C}<L<3 R_{C}$. For $L<5 \mathrm{~mm}, \mathrm{TE}_{10}$ source has higher $\eta$ for $f<f_{\text {opt. }}$. However, $\mathrm{TM}_{10}$ source operating at $f_{\text {opt }} \approx$ $1.2 \mathrm{GHz}$ results in a 55\% improvement of $\max (\eta)$ compared to $\mathrm{TE}_{10}$. For $f>f_{\mathrm{opt}}$, the decline of $\eta$ is identical for both source formulations - it is driven mainly by the propagating wave attenuation inside of lossy tissues.

A capsule shell insulates the source from lossy tissues and helps to improve the radiation performance [25]. First, we characterize the effect of a lossless shell thickness $T$ (i.e. $\sigma=$ $0 \mathrm{~S} \cdot \mathrm{m}^{-1}$ ) on $\max (\eta)$ given the shell permittivity $\varepsilon_{r}$ matches the one of the surrounding tissue (muscle). As Fig. $3 b$ shows, the radiation efficiency is proportional to $T$ for both source formulations although the effect is stronger for $\mathrm{TM}_{10}$. Obviously, the latter is due to the reduction of losses in the predominantly electric near field of the $\mathrm{TM}_{10}$ source.

Next, we evaluate the effects of dielectric loading on the radiation efficiency. We set the permittivity $\varepsilon_{r, S}$ of the capsule volume $\Omega_{S}$ proportional to $\varepsilon_{r}$ of the surrounding muscle tissue $\left(\Omega_{P}\right.$, Fig. 1) via a factor $c_{\mathrm{dl}}-$ a dielectric loading coefficient. So, $\varepsilon_{r, S}=c_{\mathrm{dl}} \varepsilon_{r}$ and $c_{\mathrm{dl}} \in\left[1 / \varepsilon_{r}, 2\right]$. When $c_{\mathrm{dl}}=1 / \varepsilon_{r}, \varepsilon_{r, S}=1$, and it implies no dielectric loading by the capsule materials (only surrounding tissues load the source [26]). For the sources considered in this study, the dielectric loading affects only the $\mathrm{TM}_{10}$ one. $\mathrm{TE}_{10}$ source remains invariant to $c_{\mathrm{dl}}$ due to its essentially magnetic near field. As Fig. 3b shows, highpermittivity capsule materials have the potential to improve the radiation efficiency of electric antennas. The thicker the shell, the stronger the effect. For a 1-mm-thick shell with $\varepsilon_{r, s}=2 \varepsilon_{r}$, $\max (\eta)=3.7 \%$ that is one order of magnitude better than state-

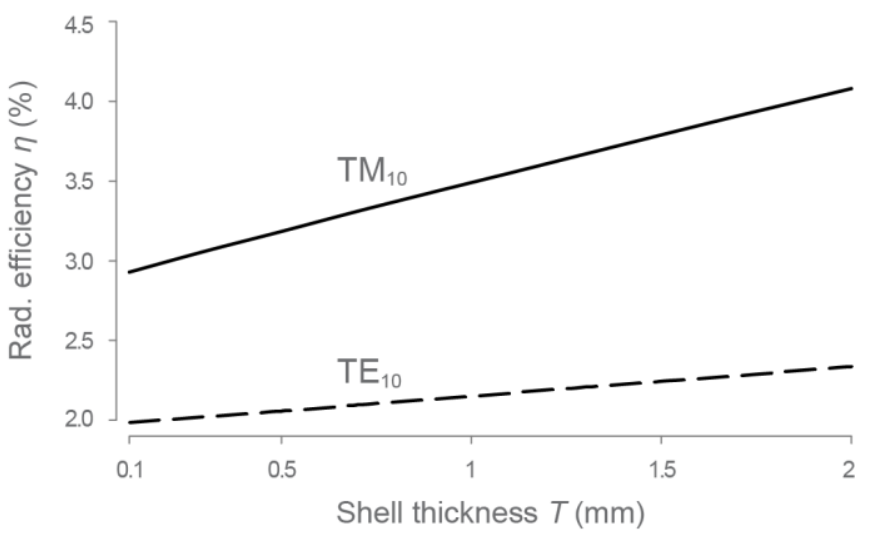

(a)

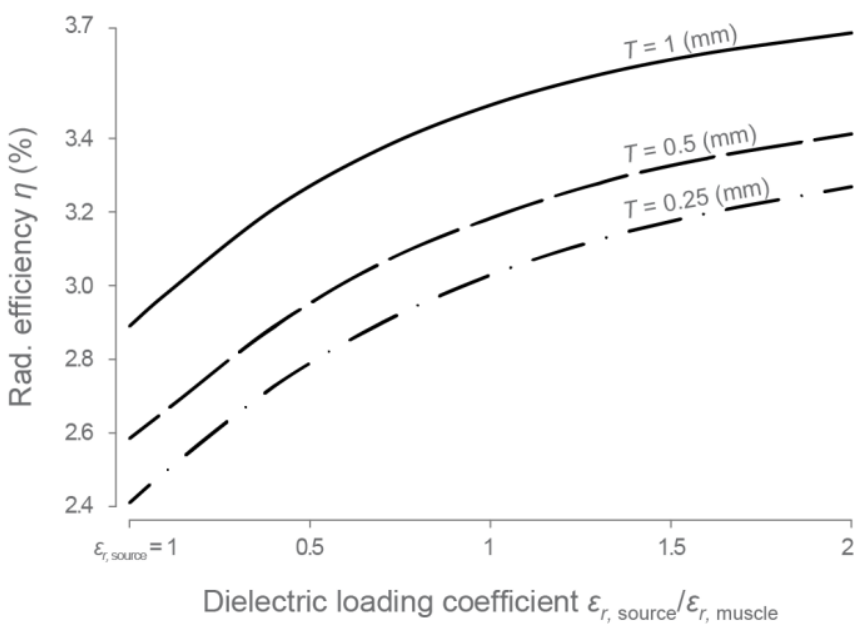

(b)

Fig. 2. Radiation efficiency as a function of (a) capsule shell thickness $T$ and (b) dielectric loading of a $\mathrm{TM}_{10}$ source $\left(\varepsilon_{r}\right.$ of $\left.\Omega_{S}\right)$. The source dimensions are $L=10 \mathrm{~mm}$ and $R_{C}=4 \mathrm{~mm}$.

of-the-art capsule antennas. Obviously, for realistic dielectricloaded capsule antennas, the improvement would be more modest considering material losses and suboptimal current distributions. 


\section{IV.CONCLUSION}

In this study, we quantified the effects of body-implantable capsule dimensions and materials on radiation performance. Electric and magnetic antennas were represented by $\mathrm{TM}_{10}$ and $\mathrm{TE}_{10}$ equivalent sources, respectively. In addition, this study confirmed previous findings that electric antennas could achieve better efficiency for in-body applications compared to the magnetic ones. For this to happen, the antenna should be sufficiently large (e.g. $a>5 \mathrm{~mm}$ ), operate close to the optimal frequency, and have a low-loss superstrate with sufficient thickness and permittivity (see Fig. 3). Note that the magnetic antennas remain more efficient when electrically small $\left(f_{0}<\right.$ $f_{\text {opt }}$ ). Future work involves investigating other antenna shapes and optimizing surface current density distributions, considering higher order modes [16], and taking into account losses due to resonance tuning [27].

\section{REFERENCES}

[1] E. Katz, Implantable Bioelectronics. Weinheim, Germany: Wiley-VCH, 2014.

[2] D. Nikolayev, M. Zhadobov, R. Sauleau, and P. Karban, "Antennas for ingestible capsule telemetry," in Advances in Body-Centric Wireless Communication: Applications and Stateof-the-Art, London, UK: IET, 2016, pp. 143-186.

[3] A. Yakovlev, S. Kim, and A. Poon, "Implantable biomedical devices: Wireless powering and communication," IEEE Commun. Mag., vol. 50, no. 4, pp. 152-159, Apr. 2012.

[4] D. Nikolayev, M. Zhadobov, P. Karban, and R. Sauleau, "Conformal antennas for miniature in-body devices: The quest to improve radiation performance," URSI Rad. Sci. Bull., vol. 2017, no. 363 , pp. 52-64, Dec. 2017.

[5] Y. Peng, K. Saito, and K. Ito, "Antenna design for impulse-radiobased wireless capsule endoscope communication systems," IEEE Trans. Antennas Propag., vol. 66, no. 10, pp. 5031-5042, Oct. 2018

[6] S. Bakogianni and S. Koulouridis, "On the design of miniature MedRadio implantable antennas," IEEE Trans. Antennas Propag., vol. 65, no. 7, pp. 3447-3455, Jul. 2017.

[7] D. Nikolayev, M. Zhadobov, L. Le Coq, P. Karban, and R. Sauleau, "Robust ultra-miniature capsule antenna for ingestible and implantable applications," IEEE Trans. Antennas Propag., vol. 65, no. 11, pp. 6107-6119, Nov. 2017.

[8] Z. Bao, Y. X. Guo, and R. Mittra, "An ultrawideband conformal capsule antenna with stable impedance matching," IEEE Trans. Antennas Propag., vol. 65, no. 10, pp. 5086-5094, Oct. 2017.

[9] Z. Bao, Y.-X. Guo, and R. Mittra, "Conformal capsule antenna with reconfigurable radiation pattern for robust communications," IEEE Trans. Antennas Propag., vol. 66, no. 7, pp. 3354-3365, Apr. 2018.

[10] J. Wang, M. Leach, E. G. Lim, Z. Wang, R. Pei, and Y. Huang, "An implantable and conformal antenna for wireless capsule endoscopy," IEEE Antenn. Wireless Propag. Lett., vol. 17, no. 7, pp. 1153-1157, Jul. 2018.

[11] W. Lei and Y. Guo, "Design of a dual-polarized wideband conformal loop antenna for capsule endoscopy systems," IEEE
Trans. Antennas Propag., vol. 66, no. 11, pp. 5706-5715, Nov. 2018.

[12] D. Nikolayev, M. Zhadobov, and R. Sauleau, "Immune-todetuning wireless in-body platform for versatile biotelemetry applications," IEEE Trans. Biomed. Circuits Syst., vol. 13, no. 2, pp. 403-412, Apr. 2019.

[13] M. S. Miah, A. N. Khan, C. Icheln, K. Haneda, and K. Takizawa, "Antenna system design for improved wireless capsule endoscope links at $433 \mathrm{MHz}$," IEEE Trans. Antennas Propag., vol. 67, no. 4, pp. 2687-2699, Apr. 2019.

[14] F. Merli, L. Bolomey, J. Zurcher, G. Corradini, E. Meurville, and A. K. Skrivervik, "Design, realization and measurements of a miniature antenna for implantable wireless communication systems," IEEE Trans. Antennas Propag., vol. 59, no. 10, pp. 3544-3555, Oct. 2011.

[15] Z. Jiang et al., "Wideband loop antenna with split ring resonators for wireless medical telemetry," IEEE Antenn. Wireless Propag. Lett., pp. 1-1, 2019.

[16] A. K. Skrivervik, M. Bosiljevac, and Z. Sipus, "Fundamental limits for implanted antennas: Maximum power density reaching free space," IEEE Trans. Antennas Propag., 2019.

[17] D. Nikolayev, W. Joseph, M. Zhadobov, R. Sauleau, and L. Martens, "Optimal radiation of body-implanted capsules," Phys. Rev. Lett., vol. 122, no. 10, p. 108101, Mar. 2019.

[18] A. Kiourti and K. S. Nikita, "A review of in-body biotelemetry devices: implantables, ingestibles, and injectables," IEEE Trans. Biomed. Eng., vol. 64, no. 7, pp. 1422-1430, Jul. 2017.

[19] S. Benaissa et al., "Experimental characterization of in-to-outbody path loss at $433 \mathrm{MHz}$ in dairy cows," Electron. Lett., vol. 55, no. 7, pp. 422-424, Apr. 2019.

[20] S. Kim, J. S. Ho, and A. S. Y. Poon, "Midfield wireless powering of subwavelength autonomous devices," Phys. Rev. Lett., vol. 110, no. 20, p. 203905, May 2013.

[21] D. Nikolayev, M. Zhadobov, P. Karban, and R. Sauleau, "Electromagnetic radiation efficiency of body-implanted devices," Phys. Rev. Applied, vol. 9, no. 2, p. 024033, Feb. 2018.

[22] S. Gabriel, R. W. Lau, and C. Gabriel, "The dielectric properties of biological tissues: III. Parametric models for the dielectric spectrum of tissues," Phys. Med. Biol., vol. 41, pp. 2271-2293, Nov. 1996.

[23] J. D. Jackson, Classical electrodynamics, 3rd ed. Hoboken, NJ: John Wiley \& Sons, 1999.

[24] P. Karban, F. Mach, P. Kůs, D. Pánek, and I. Doležel, "Numerical solution of coupled problems using code Agros2D," Computing, vol. 95, no. 1, pp. 381-408, 2013.

[25] F. Merli, B. Fuchs, J. R. Mosig, and A. K. Skrivervik, "The effect of insulating layers on the performance of implanted antennas," IEEE Trans. Antennas Propag., vol. 59, no. 1, pp. 21-31, Jan. 2011.

[26] D. Nikolayev, M. Zhadobov, and R. Sauleau, "Impact of tissue electromagnetic properties on radiation performance of in-body antennas," IEEE Antenn. Wireless Propag. Lett., vol. 17, no. 8, pp. 1440-1444, Aug. 2018 .

[27] L. Jelinek, K. Schab, and M. Capek, "Radiation efficiency cost of resonance tuning," IEEE Trans. Antennas Propag., vol. 66, no. 12, pp. 6716-6723, Dec. 2018. 